\title{
Next-Generation Sequencing-Based Molecular Diagnosis of Choroideremia
}

\author{
Kayo Shimizu $^{\mathrm{a}}$ Akio Oishi $^{\mathrm{a}}$ Maho Oishi $^{\mathrm{a}}$ Ken Ogino ${ }^{\mathrm{a}}$ \\ Satoshi Morooka ${ }^{a}$ Masako Sugahara ${ }^{a}$ Norimoto Gotoh ${ }^{b}$ \\ Nagahisa Yoshimura ${ }^{a}$ \\ ${ }^{a}$ Department of Ophthalmology and Visual Sciences, ${ }^{b}$ Center for Genomic Medicine, Kyoto \\ University Graduate School of Medicine, Kyoto, Japan
}

\section{Key Words}

Choroideremia $\cdot$ Next-generation sequencing $\cdot$ Diagnosis $\cdot$ Utility

\begin{abstract}
We screened patients with choroideremia using next-generation sequencing (NGS) and identified a novel mutation and a known mutation in the CHM gene. One patient presented an atypical fundus appearance for choroideremia. Another patient presented macular hole retinal detachment in the left eye. The present case series shows the utility of NGS-based screening in patients with choroideremia. In addition, the presence of macular hole in 1 of the 2 patients, together with a previous report, indicated the susceptibility of patients with choroideremia to macular hole.

(c) 2015 S. Karger AG, Basel
\end{abstract}

\section{Introduction}

Choroideremia is an X-linked chorioretinal degenerative disease [1]. The causative gene, mutated in this disease, was identified as the CHM gene (also known as Rab Escort Protein 1, REP-1, NM_000390) in the 1990s [2]. Although there is currently no effective treatment for the disease, gene therapy is being explored [3]. Patients suffer night blindness, visual field defect, and visual acuity impairment. The visual dysfunction often progresses to total blindness. A definite diagnosis can be made with genetic analysis. However, it is sometimes difficult to differentiate this disease from other retinal dystrophies just based on clinical features [4]. In these settings, the CHM gene mutation screening is not a practical routine test in pa-

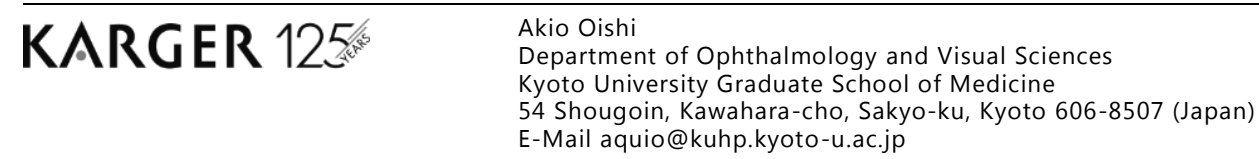


Shimizu et al.: Next-Generation Sequencing-Based Molecular Diagnosis of Choroideremia

tients with heterogeneous diseases including choroideremia, retinitis pigmentosa, cone-rod dystrophy, or other dystrophies.

Next-generation sequencing (NGS) is a genetic analysis technique that generates a large amount of DNA sequence data and enables a faster and more cost-effective sequencing of multiple genes in multiple patients than conventional sequencing [5]. We used NGS for retinal dystrophies and reported the prevalence of mutations in retinitis pigmentosa [6]. During the analysis, we additionally screened patients with other retinal dystrophies and identified a total of 2 patients with choroideremia. Herein, we describe these 2 cases and discuss the clinical relevance of comprehensive genetic analysis using NGS.

\section{Case Reports}

Case 1

A 41-year-old man was diagnosed with retinitis pigmentosa when he was 7 years old. His younger brother was also diagnosed with retinitis pigmentosa. Visual acuity was 50 $\mathrm{cm} /$ hand motion in both eyes. Fundus photography showed diffuse chorioretinal atrophy throughout the entire retina. Only small islands of visual field were preserved. Optical coherence tomography (OCT) scans showed abnormal thinning of the retina, especially in the outer retinal layers in both eyes. The left eye exhibited macular hole retinal detachment (MHRD; fig. 1). Despite the previous diagnosis of retinitis pigmentosa, fundus appearance and family history prompted a diagnosis of choroideremia. Comprehensive genetic screening using NGS was performed as previously described [6], and a novel CHM mutation (c.1718_1719delAT, p.Y573fs) was detected. The mutation was confirmed with Sanger sequencing.

\section{Case 2}

A 22-year-old man experienced night blindness when he was 3 years old and was diagnosed with retinitis pigmentosa when he was 8 years old. Pigmentary fundus abnormality was also noted in his sister. However, the diagnosis details were unknown because the examination was performed in another institution. His visual acuity was 1.5 in both eyes, but his fundus showed chorioretinal atrophy, spreading from the posterior pole, except for the macula area, to around the equatorial fundus. Ring-shaped scotoma was detected with Goldmann perimeter. OCT scan showed loss of photoreceptors (fig. 2). While the fundus appearance was atypical for choroideremia, a known CHM mutation (c.808C >T, p.R270*) [7] was detected by NGS in this case [6]. Again, the mutation was confirmed with Sanger sequencing.

\section{Discussion}

Herein, we described 2 patients with choroideremia, whose molecular diagnosis was obtained by NGS. For case 1, the diagnosis could be made with conventional sequencing, since the fundus appearance was characteristic for choroideremia. Nevertheless, direct sequencing is time- and labor-consuming for such a gene with 17 exons. Microarray-based screening can be an alternative method [8] but cannot detect novel mutations as the one detected in this case. We believe that NGS-based screening was useful in detecting the causative mutation in this particular case. 
Shimizu et al:: Next-Generation Sequencing-Based Molecular Diagnosis of Choroideremia

The second case clearly shows the utility of NGS. The fundus appearance was atypical for choroideremia. The family history (abnormal fundus in his sibling) suggested an autosomal-recessive inheritance trait. In this situation, it was difficult to suspect choroideremia based on clinical presentation. In fact, we included this patient in our previous study dealing with retinitis pigmentosa. Comprehensive screening using NGS allowed the detection of a known mutation, and the clinical diagnosis was revised to choroideremia [6].

MHRD was incidentally found in the first case. The formation of macular hole (MH) in choroideremia has previously been reported $[9,10]$. While the authors did not discuss its prevalence, they showed that 3 out of 30 patients with choroideremia had MH [9]. In the present case series, 1 out of 2 patients presented MHRD. In contrast, an OCT-based study on retinitis pigmentosa reported the prevalence of $\mathrm{MH}$ as $0.9 \%$ (3/323 patients) [11]. These data indicate that patients with choroideremia might be susceptible to MH formation. Although it is not easy to estimate the exact prevalence, $\mathrm{MH}$ formation should be carefully taken into consideration in patients with choroideremia.

The significance of molecular diagnosis in inherited retinal dystrophies has been limited because there has been no available treatment regardless of the causative gene. However, new therapeutic strategies are evolving such as gene replacement therapy for Leber congenital amaurosis [12] and choroideremia [3]. Recruiting patients, who could potentially benefit from the trial, will be crucial in the near future. NGS would serve as a tool to identify the candidate patients.

\section{Acknowledgement}

This study was supported in part by the Japan Ministry of Health, Labour and Welfare (No. 12103069), a grant-in-aid for scientific research (No. 26861445) from the Japan Society for the Promotion of Science, and the Japanese Retinitis Pigmentosa Society. The funding organizations had no role in the design or execution of this research.

\section{Statement of Ethics}

This study adheres to the tenets of the Declaration of Helsinki. The institutional review boards and the ethics committees of each institution involved approved the protocols of the study. All patients and their relatives were fully informed of the purpose and procedures of this study, and written consent was obtained from each participant.

\section{Disclosure Statement}

All authors have no conflict of interest.

\section{References}

1 Coussa RG, Traboulsi EI: Choroideremia: a review of general findings and pathogenesis. Ophthalmic Genet 2012;33:57-65.

2 Cremers FP, van de Pol DJ, van Kerkhoff LP, Wieringa B, Ropers HH: Cloning of a gene that is rearranged in patients with choroideraemia. Nature 1990;347:674-677. 
Shimizu et al:: Next-Generation Sequencing-Based Molecular Diagnosis of Choroideremia

3 MacLaren RE, Groppe M, Barnard AR, et al: Retinal gene therapy in patients with choroideremia: initial findings from a phase 1/2 clinical trial. Lancet 2014;383:1129-1137.

4 Lee TK, McTaggart KE, Sieving PA, et al. Clinical diagnoses that overlap with choroideremia. Can J Ophthalmol 2003;38:364-372, quiz 372.

5 Rabbani B, Tekin M, Mahdieh N: The promise of whole-exome sequencing in medical genetics. J Hum Genet 2014;59:5-15.

6 Oishi M, Oishi A, Gotoh N, et al: Comprehensive molecular diagnosis of a large cohort of Japanese retinitis pigmentosa and usher syndrome patients by next-generation sequencing. Invest Ophthalmol Vis Sci 2014;55:7369-7375.

7 Fujiki K, Hotta Y, Hayakawa M, et al: REP-1 gene mutations in Japanese patients with choroideremia. Graefes Arch Clin Exp Ophthalmol 1999;237:735-740.

-8 Klevering BJ, Yzer S, Rohrschneider K, et al: Microarray-based mutation analysis of the ABCA4 (ABCR) gene in autosomal recessive cone-rod dystrophy and retinitis pigmentosa. Eur J Hum Genet 2004;12:1024-1032.

-9 Zinkernagel MS, Groppe M, MacLaren RE: Macular hole surgery in patients with end-stage choroideremia. Ophthalmology 2013;120:1592-1596.

10 Shinoda H, Koto T, Fujiki K, Murakami A, Tsubota K, Ozawa Y: Clinical findings in a choroideremia patient who underwent vitrectomy for retinal detachment associated with macular hole. Jpn J Ophthalmol 2011;55:169-171.

11 Hagiwara A, Yamamoto S, Ogata K, et al: Macular abnormalities in patients with retinitis pigmentosa: prevalence on OCT examination and outcomes of vitreoretinal surgery. Acta Ophthalmol 2011;89:e122e125.

$\checkmark 12$ Cideciyan AV, Hauswirth WW, Aleman TS, et al: Human RPE65 gene therapy for Leber congenital amaurosis: persistence of early visual improvements and safety at 1 year. Hum Gene Ther 2009;20:999-1004.
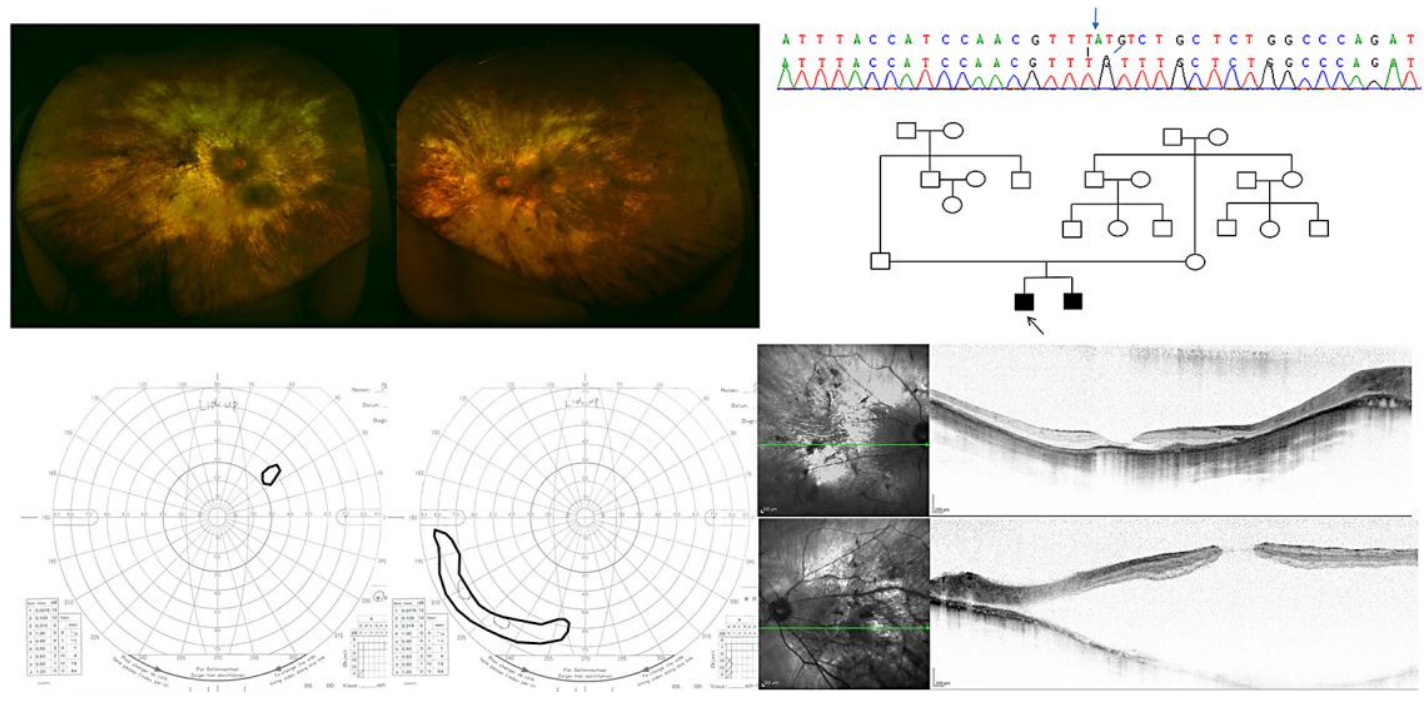

Fig. 1. Fundus photographs showing diffuse chorioretinal atrophy throughout the entire retina in both eyes (upper left). The visual field was severely impaired with only small islands remaining (lower left). Sanger sequence confirmed the 2-bp deletion (upper right, arrow). In addition, there was another single nucleotide polymorphism c.1722C $>\mathrm{T}$, which was synonymous if without a frameshift. Family history suggested autosomal recessive or X-linked inheritance trait (middle right). OCT scan showing abnormal thinning of the retina in both eyes. MHRD was noted in the left eye (lower right). 
Shimizu et al.: Next-Generation Sequencing-Based Molecular Diagnosis of Choroideremia

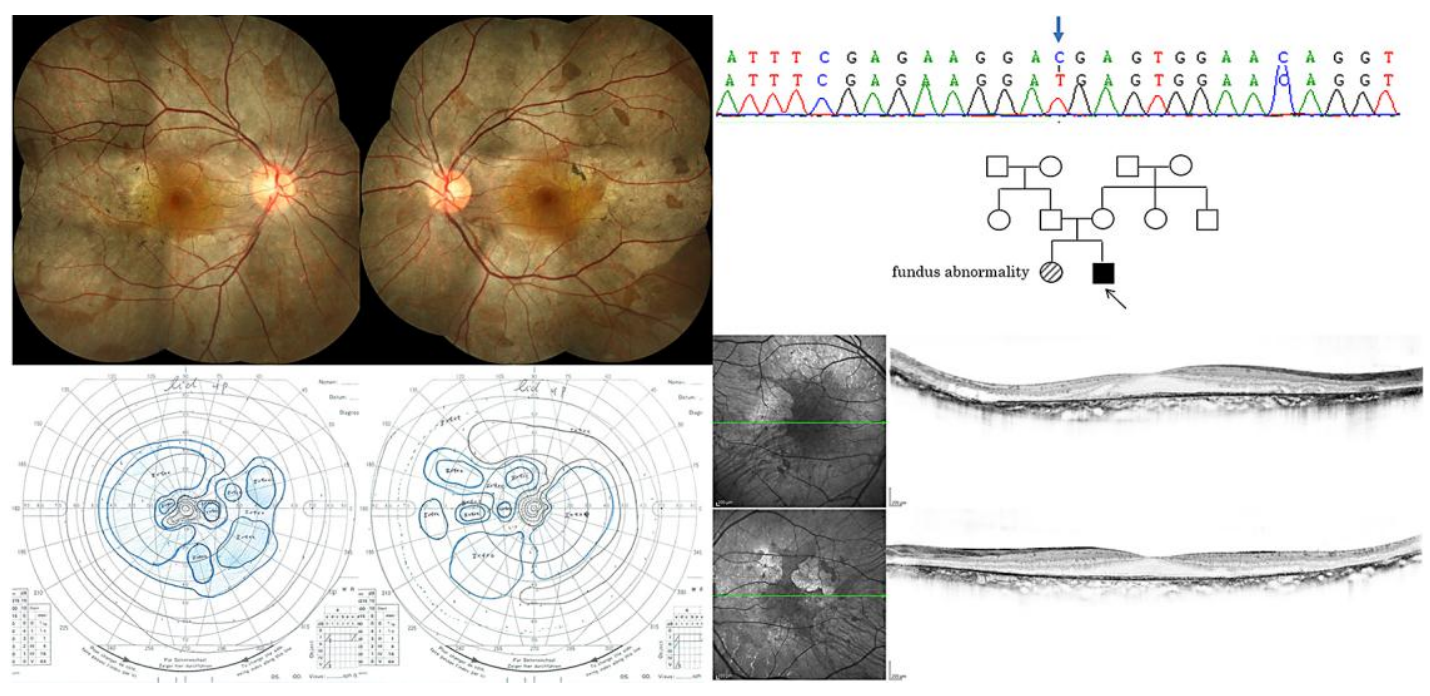

Fig. 2. Fundus photographs showing chorioretinal atrophy, spreading from the posterior pole, except for macula area, to around the equatorial fundus (upper left). The visual field showed ring-shaped scotoma (lower left). Sanger sequencing confirmed the presence of the mutation (upper right). Family history suggested autosomal recessive inheritance trait (middle right). OCT showed loss of photoreceptors in both eyes. Foveal thickness was relatively preserved (lower right). 